HNO 2014 · 62:845

DOI 10.1007/s00106-014-2939-9

Online publiziert: 18. Oktober 2014

c) Springer-Verlag Berlin Heidelberg 2014

T. Hummel

Arbeitsbereich Riechen und Schmecken, Universitäts-HNO Klinik, TU Dresden

\title{
Riechvermögen und Riechstörungen
}

Dieses Leitthemenheft befasst sich mit dem Thema „Riechen“. Was ist das Besondere an diesem Heft? Es ist verfasst von Autoren, die regelmäßig Patienten mit Riechstörungen sehen bzw. sich mit diesem Thema auseinandersetzen. Alle Autoren sind international ausgewiesene Experten auf diesem Feld, was durch entsprechende Publikationen dargestellt ist. Auf den Leser wartet also eine relativ kurze, kompetente Zusammenfassung zur Olfaktorik.

\section{》) Auf den Leser wartet eine relativ kurze Zusammenfassung zur Olfaktorik}

Was wissen Sie nach dem Lesen? Das Leitthema ist in 3 Kapitel gegliedert:

- Grundlagen des Riechvermögens,

- Diagnostik von Riechstörungen und

- Erfassung der Ursachen und Therapie von Riechstörungen.

Bei den Grundlagen des Riechvermögens wird nicht nur dargestellt, wie Riechen funktioniert, von der Nase bis zum Gehirn, sondern auch was Riechen bedeutet und was uns fehlt, wenn wir nicht mehr riechen können;

Hinsichtlich der Diagnostik von Riechstörungen geht es darum, welche Messungen bei Riechstörungen sinnvoll sind und was Riechtests leisten können.

Bei der Erfassung der Ursachen und Therapie von Riechstörungen werden verschiedene Therapieansätze erläutert, wobei insbesondere den antientzündlichen Maßnahmen sowie regenerativen Fähigkeiten des olfaktorischen Epithels besondere Bedeutung zukommt.
Wissenschaftlich gesehen ist „Riechen" immer noch ein junges Feld. Aber gerade das vorliegende Heft zeigt, dass wir viel mehr wissen, als manche glauben, v. a. was die Prognose von Riechstörungen angeht.

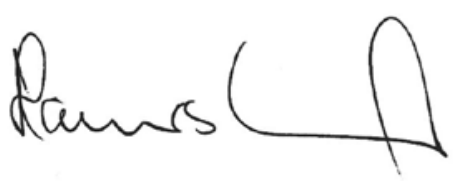

Thomas Hummel

\section{Korrespondenzadresse}

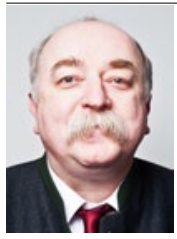

Prof. Dr. T. Hummel

Arbeitsbereich Riechen und Schmecken Universitäts-HNO Klinik TU Dresden Fetscherstr. 74, 01307 Dresden thummel@ mail.zih.tu-dresden.de

\section{Einhaltung ethischer Richtlinien}

Interessenkonflikt. T. Hummel gibt an, dass kein Interessenkonflikt besteht.

Dieser Beitrag beinhaltet keine Studien an Menschen oder Tieren. 